\title{
OPEN Ag nanoparticles immobilized on new mesoporous triazine-based carbon (MTC) as green and recoverable catalyst for reduction of nitroaromatic in aqueous media
}

\begin{abstract}
Narges Vahedi-Notash, Majid M. Heravi ${ }^{\bowtie}$, Ali Alhampour \& Pourya Mohammadi
In this research, we reported an effective method for the synthesis of a new mesoporous triazinebased carbon (MTC) substrate and its application as the green and recoverable catalyst in the synthesis of organic compounds. The porous carbon acted as a substrate for silver active species after its surface modification by chloroacetonitrile (Ag@MTC).The Ag@MTC nanocatalyst was characterized by several techniques namely, Fourier-transform infrared spectroscopy, field emission scanning electron microscopy with energy dispersive spectroscopy, X-ray diffraction, transmission electron microscopy, Brunauer-Emmett-Teller surface area analysis, and inductively coupled plasma. The Ag@MTC catalyst was applied for the reduction of nitroaromatic compounds in aqueous media by using $\mathrm{NaBH}_{4}$ (reducing agent) at room temperature. This nanocatalyst can be readily recovered and recycled for at least nine runs without a notable decrease in its efficiency. Catalytic efficiency studies exhibited that Ag@MTC nanocatalyst had good activity towards reduction reactions.
\end{abstract}

Nitrophenol compounds are categorized by the United States Environmental Protection Agency (USEPA) as the main water contaminations $\mathrm{s}^{1,2}$ owing to their great toxicity and stability to chemical treatments and microbial degradation. Nitro compounds as the main based materials are broadly applied in medicine, ceramics, textile, cosmetics, paper, food processing, and printing industries ${ }^{3,4}$. Therefore, these compounds can be found at great concentrations in their effluent and severely influence aquatic life and human health ${ }^{5-9}$. Consequently, based on the standards presented by the USEPA ${ }^{10,11}$ it is required to decrease their concentrations under 10 ppm, before being released into the media.

The common wastewater treatment techniques including chemical coagulation, adsorption, precipitation, and reverse osmosis have been utilized for the removal of nitro compounds. Nevertheless, these techniques cannot be satisfactory and efficient in the decolonization of nitro compounds owing to their difficulties such as high cost and production of dangerous by-products ${ }^{12-18}$. Hence, it is essential to utilize environmentally friendly and very effective techniques for the removal of nitro compounds. One of the methods in wastewater treatment that has attracted notable attention recently is the utilization of noble metal based catalyst such as $\mathrm{Pt}, \mathrm{Pd}, \mathrm{Ag}$, and $\mathrm{Au}$ nanoparticles owing to their high surface area, great chemical efficiency and reactivity ${ }^{19}$. In this respect, a broad variety of catalytic methods has been applied for the selective reduction of nitroaromatic compounds by using low-cost metal nanoparticles and alternative hydrogen sources such as $\mathrm{H}_{3} \mathrm{NBH}_{3}, \mathrm{NaBH}_{4}, \mathrm{NH}_{2} \mathrm{NH}_{2} \cdot \mathrm{H}_{2} \mathrm{O}$, etc. $^{20-24}$. Among the noble metal nanoparticles, silver nanoparticles have attracted significant attention as a catalyst for hydrogenation reactions (hydrogenation of azo dyes and nitroaromatics) and oxidation reactions (selective catalytic oxidation of ammonia, ethylene epoxidation, etc.) ${ }^{25}$. Howsoever, nanoparticles with large surface areas have an intrinsic trend to decrease their energy via agglomeration ${ }^{26}$. To intercept the process, the nanoparticles are frequently capped with numerous stabilizers such as surfactants ${ }^{27}$, ligands ${ }^{28}$, dendrimers ${ }^{29}$ and polymers ${ }^{30-32}$. But these compounds seriously restrict the availability of the reactant particle to the catalyst 
surface and so diminishing the catalytic activity. To defeat this problem, nanoparticles are stabilized on numerous solid supports this issue improves the availability of their surface, and the catalyst recovery. Using the carbon mesoporous materials are widely applied as catalytic support materials due to inexpensive, great specific surface area, simple synthesis process, good chemical and thermal stability, and tunable morphological structures ${ }^{33,34}$. Modifying the surface of various carbon materials such as graphite, graphene, porous carbon, carbon nanotubes, nanowires, and fullerenes can chemically create new applications by improving the properties. Several atoms such as nitrogen, oxygen, sulfur, boron, phosphorus, and halogens such as fluorine, chlorine, and iodine are used as surface modifiers of carbon materials and affect surface properties. One of the most important heteroatoms used to modify carbon surfaces is the nitrogen atom. The presence of nitrogen on carbon surfaces improves surface properties, which can be attributed to increased temperature stability, increased chemical stability, increased electrical conductivity and electronic properties adjustment, active sites on carbon substrates as well as the dispersion of metal components on carbon substrates ${ }^{35-37}$.

In this study, we explained the synthesis of functionalized carbon mesoporous material supported by silver nanoparticles (Ag@MTC) as a recoverable nanocatalyst and it was also investigated for its catalytic activity for the reduction of nitro aromatic compounds.

\section{Experimental}

Chemicals. The compounds used in the catalyst synthesis as well as in the chemical reactions i.e. chloroacetonitrile $\left(\mathrm{ClCH}_{2} \mathrm{CN}, 99 \%\right)$, D (+)-Glucose monohydrate $\left(\mathrm{C}_{6} \mathrm{H}_{12} \mathrm{O}_{6} \cdot \mathrm{H}_{2} \mathrm{O}\right)$, silver nitrate $\left(\mathrm{AgNO}_{3}\right.$, 99\%), sodium hydroxide $(\mathrm{NaOH}, 98 \%)$, sodium borohydride $\left(\mathrm{NaBH}_{4}, 98 \%\right)$, were prepared from Merck, Germany, Sigma Aldrich, and Fluka chemical companies. The precursor materials and solvents were all highly purified, without the need for further purification.

Apparatus. Scanning electron microscopy (SEM) images were acquired by a SEM microscope (Tescan VEGA3, USA). X-ray diffraction (XRD) patterns were recorded on a PW 1800 X-ray diffractometer (Philips, Netherlands) with $\mathrm{Cu}-\mathrm{K}_{\alpha}$ radiation. The IR spectra of the samples were determined using the FT-IR-8400S Spectrometer (SHIMADZU, Japan). Transmission electron microscopy images were taken using a transmission microscope (Philips CM30, 300KV). $\mathrm{N}_{2}$ sorption analysis was performed on a volumetric adsorption analyzer (BELSORP MINI II). The specific surface areas were acquired by using the Brunauer-Emmett-Teller (BET) method.

Synthesis of Ag@MTC nanocatalyst. The synthesis steps of silver nanoparticle catalysts on the carbon mesoporous substrate (Ag@MTC) were described below.

First, a mixture of glucose $(2.0 \mathrm{~g})$ with sodium hydroxide $(2.7 \mathrm{~g})$ was prepared in $20 \mathrm{~mL}$ distilled water and stirred for $12 \mathrm{~h}$ in an ice bath. Then a solution of $3.0 \mathrm{~g}$ chloroacetonitrile in $4.0 \mathrm{~mL}$ of isopropanol was prepared and added to the first mixture and stirred at $65^{\circ} \mathrm{C}$ for $12 \mathrm{~h}$. After this time, the reaction was complete by addition ethanol (70\%) to the above solution and the product was separated by centrifugation and washed three times with ethanol (70\%). Finally, $5.0 \mathrm{~mL}$ absolute ethanol was added to the above mixture and dried for $12 \mathrm{~h}$ at $65^{\circ} \mathrm{C}$.

Subsequently, the cyanomethyl glucose was thoroughly mixed with zinc chloride (8:1 molar ratio) and the mixture was heated for $40 \mathrm{~h}$ at $400{ }^{\circ} \mathrm{C}$. After cooling down to room temperature, the mixture was rinsed with distilled water and stirred for $24 \mathrm{~h}$ in hydrochloric acid solution $(1 \mathrm{M})$ to remove excess zinc chloride, then the obtained black powder was washed with water, tetrahydrofuran, and acetone to remove all salts and the excess hydrochloric acid, then the product was dried in a vacuum oven at $100^{\circ} \mathrm{C}^{38}$.

In order to synthesize silver nanoparticles on carbon mesoporous substrate, $470 \mathrm{mg}$ of the glucose mesoporous substrate was first dispersed in $100 \mathrm{~mL}$ of water. Next, $4.72 \mathrm{~mL}$ of silver nitrate solution $\left(10 \mathrm{mg} \mathrm{mL}^{-1}\right)$ was added to the first mixture under ultrasonication and then $15 \mathrm{~mL}$ of $210 \mathrm{mg} \mathrm{NaBH}_{4}$ aqueous solution was gently added to the above mixture and stirred for $12 \mathrm{~h}$ at room temperature. Ag@MTC nanocatalyst was separated by centrifugation and dried at $30^{\circ} \mathrm{C}^{39}$.

Catalytic reduction of nitrophenols. Catalytic reduction reactions of nitro compounds in the presence of $\mathrm{NaBH}_{4}$ were carried out in the aqueous solution at the intended temperature. In a typical experiment, a $3.0 \mathrm{~mL}$ of nitroaromatic solution $(1.0 \mathrm{mmol})$ was prepared and $30 \mathrm{mg}$ of Ag@MTC catalyst was dispersed in this solution under $75^{\circ} \mathrm{C}$, and this mixture was continuously stirred. Next, $5.0 \mathrm{mmol}$ freshly prepared $\mathrm{NaBH}_{4}$ solution was added to the above mixture under stirring. The progress of the reaction was controlled by a thin layer chromatography (TLC, normal hexane-ethyl acetate as solvent). After the end of the reaction, the catalyst was collected by centrifugation and the products were purified by short column chromatography over silica gel.

\section{Result and discussion}

Characterization of Ag@MTC nanocomposite. FTIR spectral analysis. For a more detailed study of the structure of the prepared nanocatalysts, the FT-IR spectra of these materials were investigated in all three stages of (a) glucose functionalized with cyanide group (Gol-CN), (b) MTC, and (c) Ag@MTC. In the spectrum (a) of Fig. 1, the peak at the region of $2327 \mathrm{~cm}^{-1}$ was related to the stretching vibration of the cyanide group and this peak showed the CN groups in the glucose structure. In the spectrum (b) of MTC, the peaks observed at $1390 \mathrm{~cm}^{-1}$ and $1606 \mathrm{~cm}^{-1}$ were ascribed to the stretching vibration of $\mathrm{C}-\mathrm{N}$ and $\mathrm{C}=\mathrm{N}$, respectively. In addition, the lack of the $-\mathrm{CN}$ group at $2327 \mathrm{~cm}^{-1}$ obviously indicated the successful formation of triazine rings. Finally, with the addition of silver nanoparticles to the MTC surface, there was no significant change in the spectrum (c). 


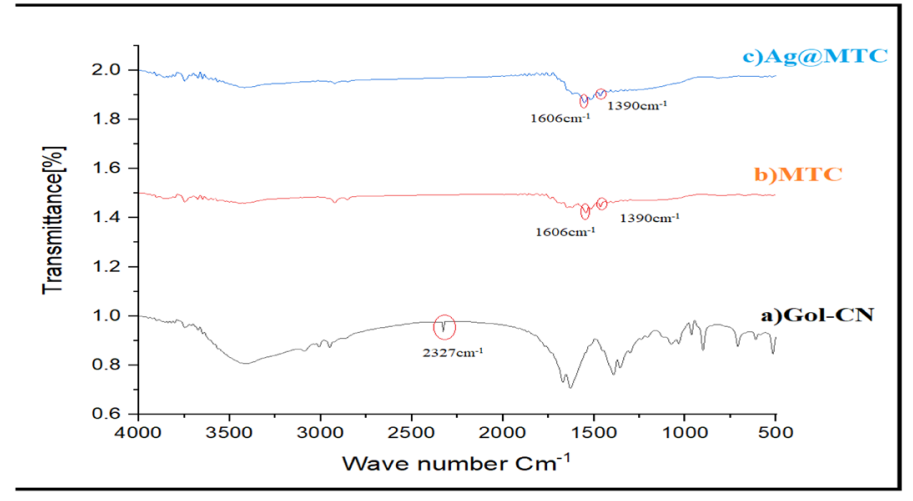

Figure 1. FTIR spectra of (a) Gol-CN, (b) MTC, and (c) Ag@MTC.

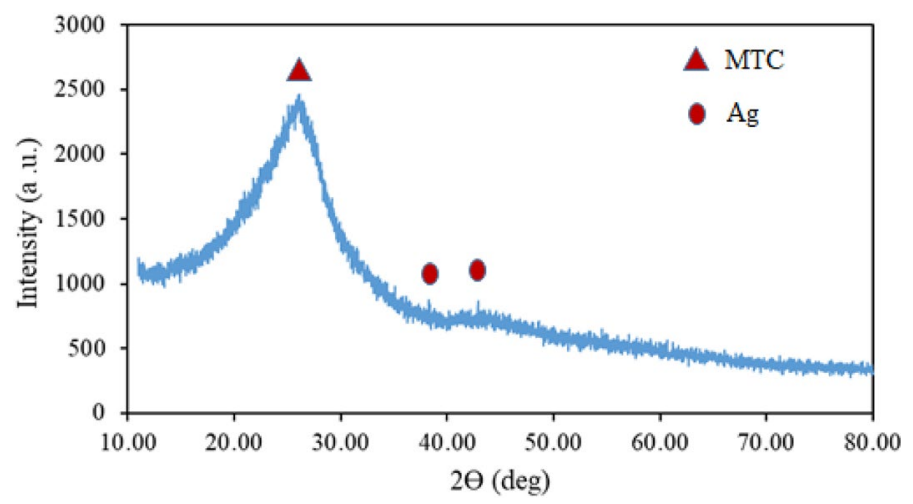

Figure 2. XRD pattern of Ag@MTC nanocomposite.

XRD patterns of the prepared nanocatalyst. In amorphous materials, the X-rays are scattered in different directions, causing a large bump and are distributed over a wide range of $2 \Theta$ rather than narrow peaks. The XRD pattern was shown in Fig. 2. As can be seen in the spectrum, no extra peak due to another phase in the sample was observed. There are two weak peaks at $2 \Theta=38.1$ and 44.2 that related to the Ag crystals. Also, a broad peak at $2 \theta=20-30$ demonstrated that the sample was amorphous and had no crystalline structure, this peak related to the MCT.

FESEM and TEM images of Ag@MTC. SEM (Fig. 3) and TEM (Fig. 4) images were applied to study the texture and morphology of the prepared nanocatalyst. Figure 3 showed the porosity of the substrate and catalyst structure. The silver nanoparticles were also clearly visible in the image and were dispersed uniformly on the MTC surface and completely covered the whole surface of this support. The uniform dispersion of Ag nanoparticles on the MTC support was also approved by the TEM image. It could be observed that Ag nanoparticles had an almost spherical structure with an average diameter of approximately $16-22 \mathrm{~nm}$.

Additionally, EDX analysis of the Ag@MTC nanocatalyst confirmed the existence of carbon, nitrogen, and silver elements. The weight \% of C, N, and Ag was confirmed in the range of 64.33, 28.93, and 6.74, respectively, as corresponded with ICP result. The mapping tests indicated that these elements in the nanocomposite were dispersed uniformly (Figs. 5, 6).

$\mathrm{N}_{2}$ absorption-desorption study. The $\mathrm{N}_{2}$ absorption-desorption isotherm of the Ag@MTC nanocatalyst was given in Fig. S1. The surface area of MTC and Ag@MTC samples were obtained about $170.22 \mathrm{~m}^{2} \mathrm{~g}^{-1}$ and 117.29 $\mathrm{m}^{2} \mathrm{~g}^{-1}$, respectively. The BJH pore volume of the MTC and Ag@MTC samples were about 0.37 and $0.12 \mathrm{~cm}^{3} \mathrm{~g}^{-1}$, respectively, and this decrease in pore volume was due to the deposition of Ag nanoparticles on the MTC surface. In addition, the BJH pore sizes of MTC and Ag@MTC samples were about $11.76 \mathrm{~nm}$ and $9.19 \mathrm{~nm}$, respectively.

ICP study. ICP analysis was used to measuring the content of silver metal present in the catalytic sample. According to the results of ICP analysis in Table 1, the content of silver loaded on the substrate was $6.7 \%$. This was consistent with the results of the EDX analysis.

Optimization of nitro reduction reaction conditions. To optimize the reaction conditions of nitro compounds using the Ag@MTC catalyst, the reduction reaction of 4-nitro aniline (4-NA) was considered as a model reac- 


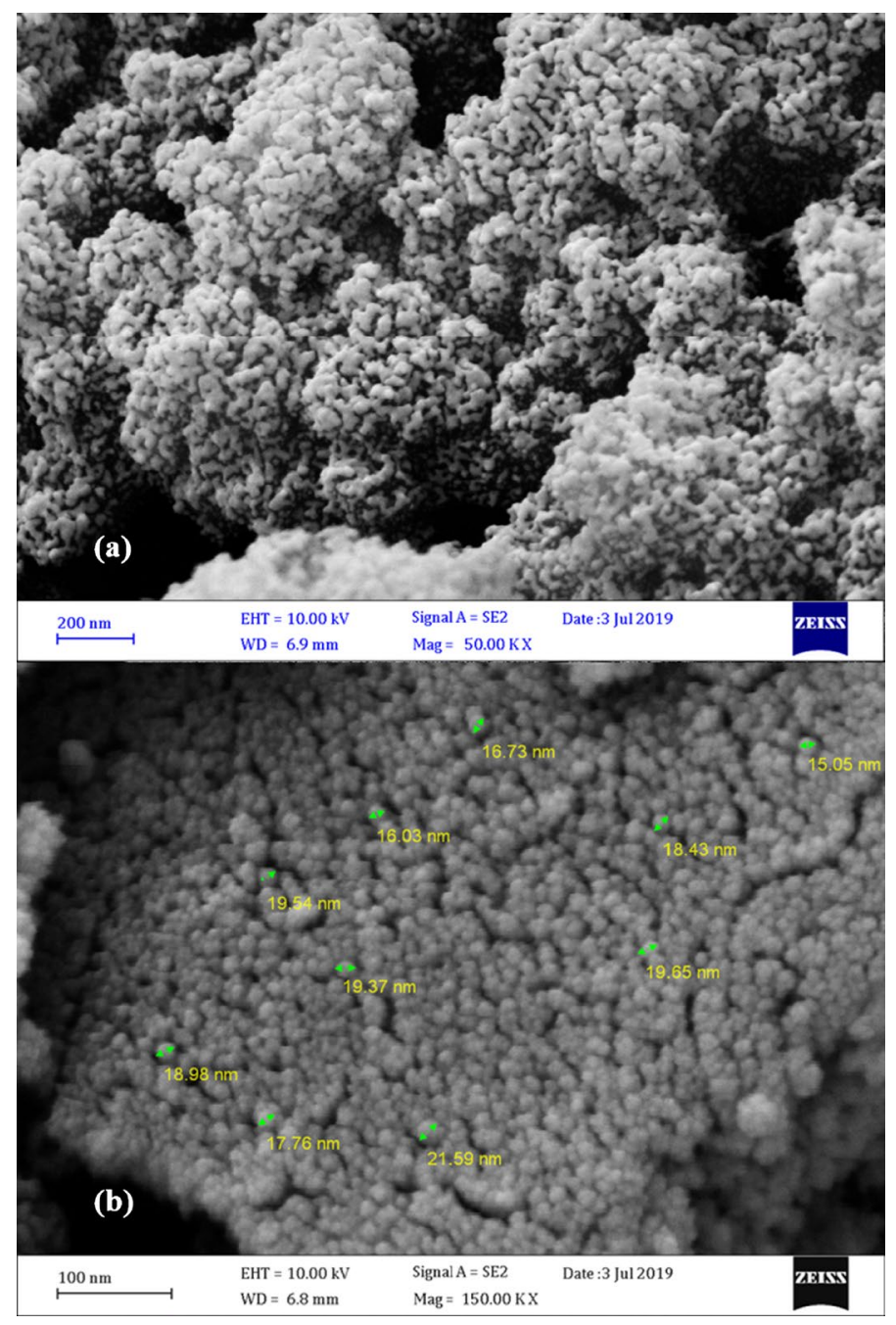

Figure 3. FESEM images of (a) MTC, (b) Ag@MTC.

tion. Therefore, the amount of nanocatalyst, the type of solvent, the type of reduction agent, and temperature were optimized.

Effect of catalyst amount on the reduction of 4-NA. In this catalysis process, the yield of reaction generally increases with increasing the amount of the Ag@MTC nanocatalyst. The yield of the reaction was considered by varying the catalyst amount from 0 to $40 \mathrm{mg}$ while the other factors were kept constant. The results were summarized in Table S1. According to the results, the reduction reaction did not occur in the absence of the Ag@MTC nanocatalyst after $180 \mathrm{~min}$. Using the smaller amounts of catalysts of 10 and $20 \mathrm{mg}$, the reduction reaction yields of 4 -NA were found to be $100 \%$ after 90 and 70 min respectively. With the increasing catalyst amount (30 mg), the yield of the reaction was obtained $100 \%$ after $20 \mathrm{~min}$. Therefore, $30 \mathrm{mg}$ was determined as the optimal amount of nanocatalyst. The reaction in the presence of the catalyst alone was tested and no progress observed.

The effect of solvents. After investigating the appropriate amount of catalyst, the next step in optimizing the reaction conditions is to investigate the effect of the solvent in the reaction progress. Hence, the effect of several solvents on the reduction reaction was presented in Table S2. As can be understood from these results, water was the best solvent for this reaction. Reaction with water/ethanol (1:1) solvent had a good yield but the choice of water was more favorable in terms of green chemistry. On the other hand, the ethanol solvent did not have a good yield, so the reaction with the water solvent was selected at $75^{\circ} \mathrm{C}$.

Effect of $\mathrm{NaBH}_{4}$ concentration for reduction of 4-NA. The $\mathrm{NaBH}_{4}$ was used as a reducing agent, this reductant cannot alone the nitro compounds reduce. So needs that the catalyst was applied. The yields of the reduction reaction were obtained at a varying concentration of $\mathrm{NaBH}_{4}$, retaining the other parameters constant. It was found that with enhancing $\mathrm{NaBH}_{4}$ concentration, the yields were increased and after a determined concentration of $\mathrm{NaBH}_{4}(5 \mathrm{mmol})$, the yields were obtained $100 \%$ after $20 \mathrm{~min}$. According to the results shown in Table S3, 


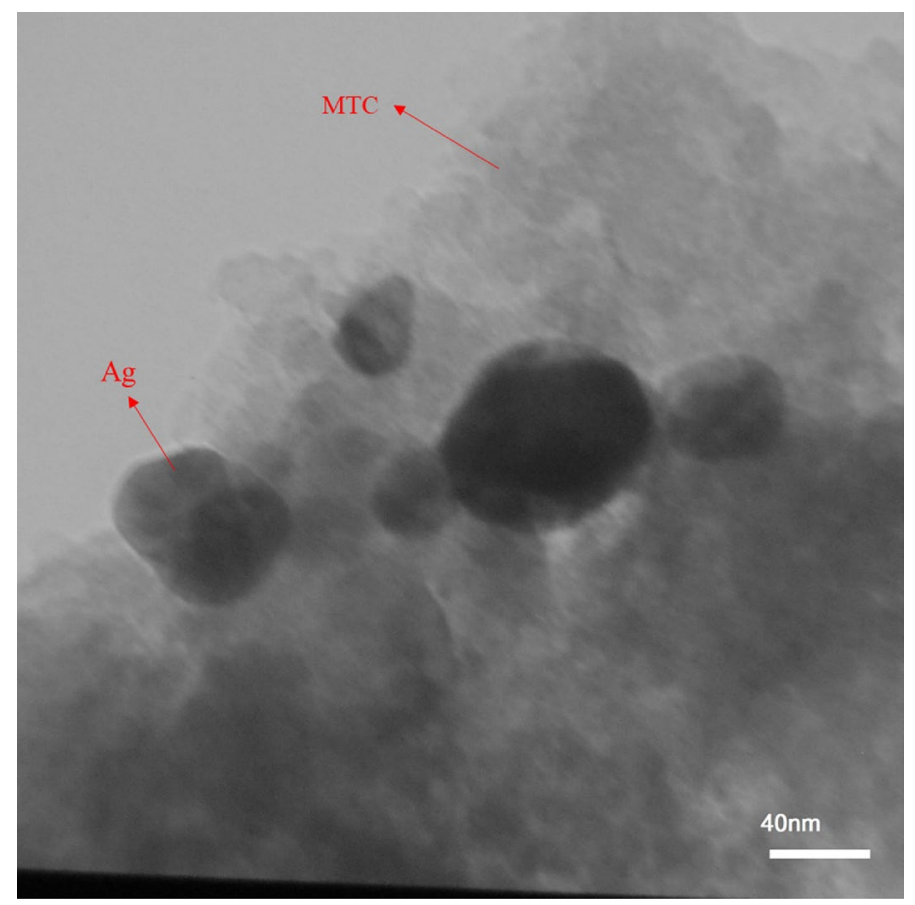

Figure 4. TEM of Ag@MTC.
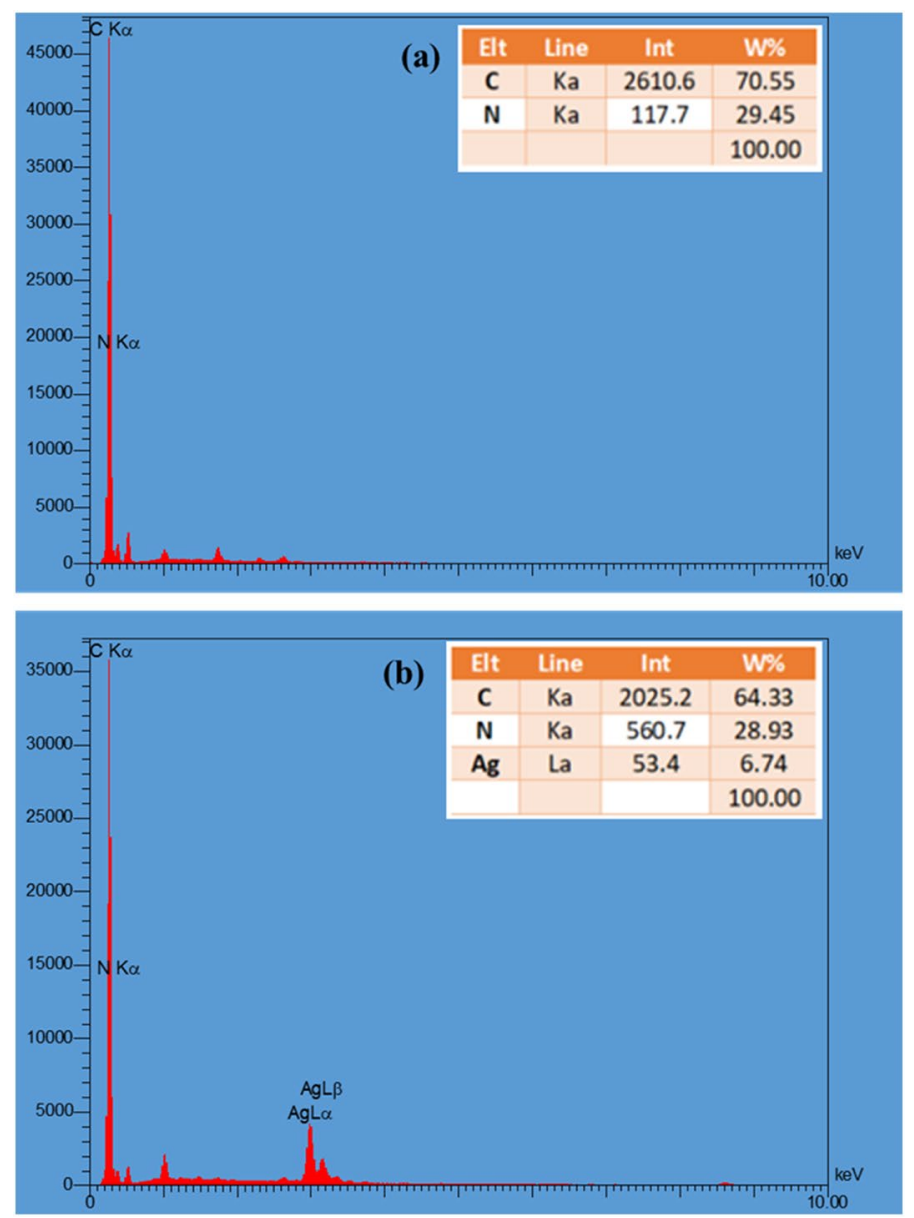

Figure 5. EDX analysis of (a) MTC, and (b) Ag@MTC. 

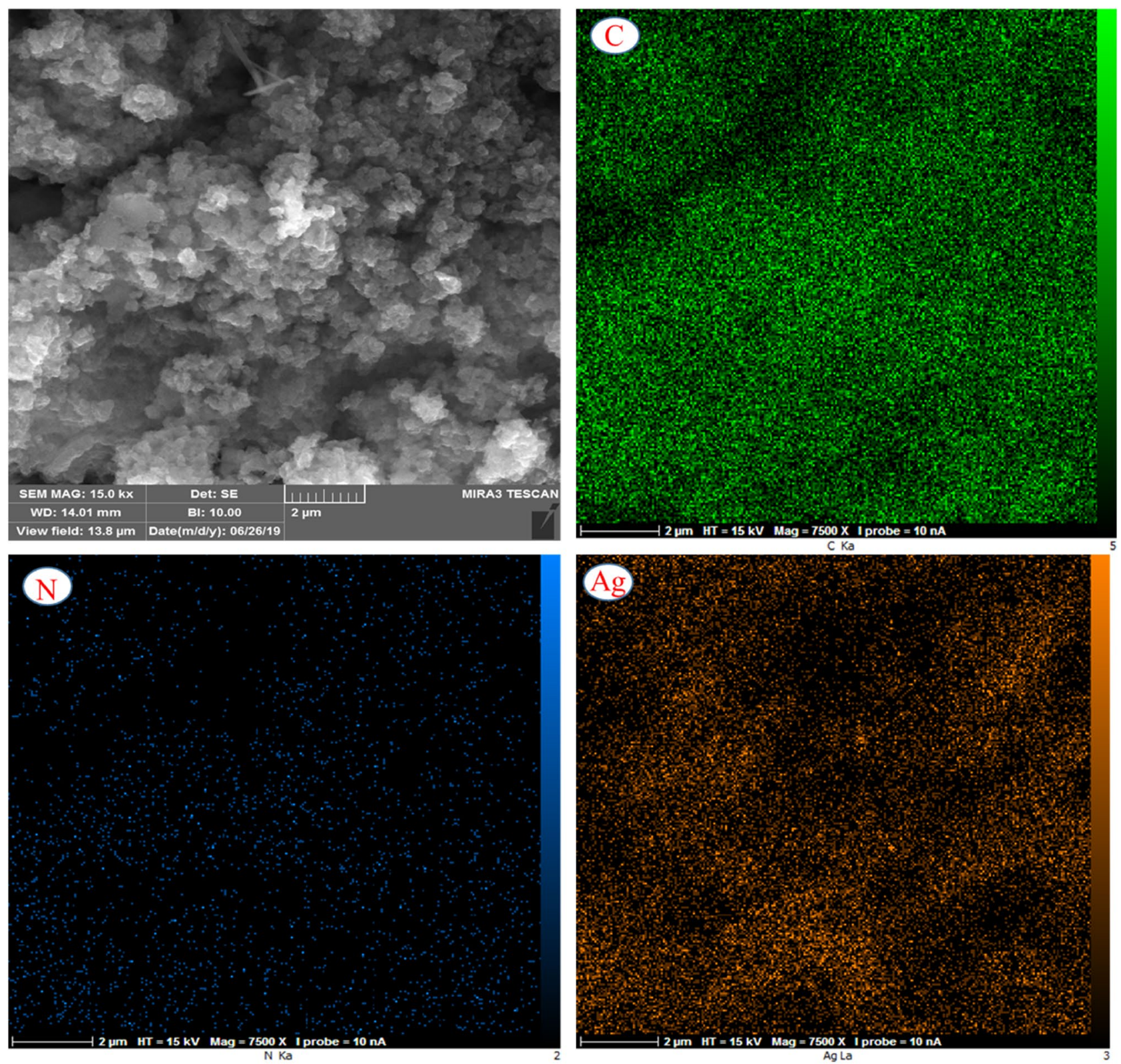

Figure 6. Mapping images of Ag@MTC.

\begin{tabular}{|l|l|}
\hline Sample & Ag content $(\mathbf{w t} \%)$ \\
\hline Ag@MTC & 6.7 \\
\hline
\end{tabular}

Table 1. ICP result of Ag@MTC.

the optimal amount for the $\mathrm{NaBH}_{4}$ was $5 \mathrm{mmol}$. The reaction in the presence of $\mathrm{NaBH}_{4}$ alone was tested and no progress observed.

Effect of temperature on the reduction of 4-NA. The reaction temperature represented a very significant role in the reduction of 4 -NA. The reaction temperatures were varied from 65 to $95^{\circ} \mathrm{C}$ while maintaining the other factors constant. The results were given in Table S4. An upsurge in reaction temperature led to obtain the higher yield. This table showed that the optimum temperature for this reaction was $75^{\circ} \mathrm{C}$.

Synthesis of nitroaromatic derivatives. The selectivity of a catalytic method possessed an essential role in identifying the conformity of a catalytic process. To investigate this scope, Ag@MTC catalyst was applied for the selective reduction of a diversity of nitroaromatic materials under the optimum conditions. The results were 


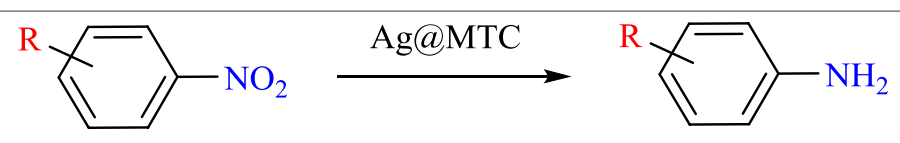

\begin{tabular}{|c|c|c|c|c|}
\hline Entry & Product & Time (min) & Yield (\%) & Melting point (Abs./ Lit.) [34] \\
\hline 1 & & 20 & 100 & $146-148 / 145-147$ \\
\hline 2 & & 40 & 100 & $103 / 102-104$ \\
\hline 3 & & 40 & 100 & Oil/6 \\
\hline 4 & & 120 & 85 & Oil/Oil \\
\hline 5 & & 50 & 80 & $187-190 / 186-190$ \\
\hline 6 & & 47 & 100 & $79-81 / 77-79$ \\
\hline 7 & & 55 & 100 & Oil/28-30 \\
\hline 8 & & 240 & 98 & $187-189 / 187-189$ \\
\hline 9 & & 45 & 86 & $95-97 / 94-98$ \\
\hline 10 & & 35 & 82 & Oil/34-37 \\
\hline
\end{tabular}

Table 2. Reduction of aromatic nitro compounds in the presence of Ag@MTC.

presented in Table 2. The efficiency of the catalyst indicated the completion of the reduction reactions in short times. Regardless of the presence of several functionalities in nitrobenzene derivatives, the nanocatalyst exhibited good yield $(80-100 \%)$ and produced the amino products. It's interesting that under the optimum conditions, hydrazine, aldehyde, carboxylic acid, and keto groups were also endured. According to the nitroaromatic compounds with electron-donating substituents, the reduction reaction was desirable and performed in shorter reaction times. Therefore, the use of cost-effective, Ag@MTC nanocatalyst for the reduction of nitro compounds was effective.

Catalyst reusability. Recyclability of catalysts is an essential parameter influencing the suitable application of the catalytic system. In order to examine the recyclability of the Ag@MTC, at the completion of the reaction, the catalyst was separated by centrifuge from the reaction mixture and reutilized in the subsequent run. The 
obtained results revealed that the synthesized nanocatalyst could be applied at least 6 consecutive runs without a notable reduction in catalytic performance (Fig. S2). (Yield 100 to $90 \%$ and Time 15 to $16 \mathrm{~min}$ ).

\section{Conclusions}

In summary, we described a facile and effective process for the synthesis of Ag@MTC nanocatalyst. The prepared Ag@MTC demonstrated good catalytic performance for the reduction of nitroaromatic compounds and possessed desirable reusability. This study hence not only represented a practical method for facile preparation of Ag@MTC but also this nanocatalyst was successfully applied for the reduction of nitro compounds. The advantages of this catalyst include low cost, short reaction time, low reaction temperature, recyclability without reduced catalytic activity and non-toxicity.

Received: 24 May 2020; Accepted: 24 July 2020

Published online: 09 November 2020

\section{References}

1. Feng, J. et al. $\mathrm{CuFe}_{2} \mathrm{O}_{4}$ magnetic nanoparticles: A simple and efficient catalyst for the reduction of nitrophenol. Chem. Eng. J. 221, 16-24 (2013).

2. Sreekanth, D., Sivaramakrishna, D., Himabindu, V. \& Anjaneyulu, Y. Thermophilic degradation of phenolic compounds in lab scale hybrid up flow anaerobic sludge blanket reactors. J. Hazard. Mater. 164(2-3), 1532-1539 (2009).

3. Banat, I. M., Nigam, P., Singh, D. \& Marchant, R. Microbial decolorization of textile-dyecontaining effluents: A review. Bioresour. Technol. 58(3), 217-227 (1996).

4. Martínez-Huitle, C. A. \& Brillas, E. Decontamination of wastewaters containing synthetic organic dyes by electrochemical methods: A general review. Appl. Catal. B 87(3-4), 105-145 (2009).

5. Sahu, K., Satpati, B. \& Mohapatra, S. Facile synthesis and phase-dependent catalytic activity of cabbage-type copper oxide nanostructures for highly efficient reduction of 4-nitrophenol. Catal. Lett. 149(9), 2519-2527 (2019).

6. Acosta, B., Evangelista, V., Miridonov, S., Fuentes, S. \& Simakov, A. The Decoration of Gold Core in Au@ZrO nanoreactors with trace amounts of Pd for the effective reduction of 4-nitrophenol to 4-aminophenol. Catal. Lett. 149(6), 1621-1632 (2019).

7. Gu, S. et al. Ligand-free gold nanoparticles as a reference material for kinetic modelling of catalytic reduction of 4-nitrophenol. Catal. Lett. 145(5), 1105-1112 (2015).

8. Vickers, N. J. Animal communication: When i'm calling you, will you answer too?. Curr. Biol. 27(14), R713-R715 (2017).

9. Mishra, K. P. \& Gogate, P. R. Intensification of sonophotocatalytic degradation of $p$-nitrophenol at pilot scale capacity. Ultrason. Sonochem. 18(3), 739-744 (2011).

10. WHO/UNICEF Joint Water Supply, Sanitation Monitoring Programme. Progress on Drinking Water and Sanitation: 2014 update (World Health Organization, 2014).

11. Deblonde, T., Cossu-Leguille, C. \& Hartemann, P. Emerging pollutants in wastewater: A review of the literature. Int. J. Hyg. Environ. Health 214(6), 442-448 (2011).

12. Ahn, W. Y., Sheeley, S. A., Rajh, T. \& Cropek, D. M. Photocatalytic reduction of 4-nitrophenol with arginine-modified titanium dioxide nanoparticles. Appl. Catal. B 74(1-2), 103-110 (2007).

13. Kurtan, U., Baykal, A. \& Sözeri, H. Recyclable $\mathrm{Fe}_{3} \mathrm{O}_{4} @$ Tween20@Ag nanocatalyst for catalytic degradation of azo dyes. J. Inorg. Organomet. Polym. Mater. 25(4), 921-929 (2015).

14. Whang, T. J., Hsieh, M. T. \& Chen, H. H. Visible-light photocatalytic degradation of methylene blue with laser-induced Ag/ZnO nanoparticles. Appl. Surf. Sci. 258(7), 2796-2801 (2012).

15. Tripathi, K. M., Tyagi, A., Ashfaq, M. \& Gupta, R. K. Temperature dependent, shape variant synthesis of photoluminescent and biocompatible carbon nanostructures from almond husk for applications in dye removal. RSC Adv. 6(35), 29545-29553 (2016).

16. Park, S. J. et al. Visible-light photocatalysis by carbon-nano-onion-functionalized ZnO tetrapods: Degradation of 2,4-dinitrophenol and a plant-model-based ecological assessment. NPG Asia Mater. 11(1), 1-3 (2019).

17. Abu-Danso, E. et al. Synthesis of clay-cellulose biocomposite for the removal of toxic metal ions from aqueous medium. J. Hazard. Mater. 381, 120871 (2020).

18. Singh, A., Bhati, A., Khare, P., Tripathi, K. M. \& Sonkar, S. K. Soluble graphene nanosheets for the sunlight-induced photodegradation of the mixture of dyes and its environmental assessment. Sci. Rep. 9(1), 1-2 (2019).

19. Takenaka, Y., Kiyosu, T., Choi, J. C., Sakakura, T. \& Yasuda, H. Selective synthesis of $N$-aryl hydroxylamines by the hydrogenation of nitroaromatics using supported platinum catalysts. Green Chem. 11(9), 1385-1390 (2009).

20. Zhao, Z., Yang, H., Li, Y. \& Guo, X. Cobalt-modified molybdenum carbide as an efficient catalyst for chemoselective reduction of aromatic nitro compounds. Green Chem. 16(3), 1274-1281 (2014).

21. Mohan, M., Mohan, N. \& Chand, D. K. Self-assembled gold nanofilms as a simple, recoverable and recyclable catalyst for nitroreduction. J. Mater. Chem. A 3(42), 21167-21177 (2015).

22. Vasilikogiannaki, E., Gryparis, C., Kotzabasaki, V., Lykakis, I. N. \& Stratakis, M. Facile reduction of nitroarenes into anilines and nitroalkanes into hydroxylamines via the rapid activation of ammonia borane complex by supported gold nanoparticles. Adv. Synth. Catal. 355(5), 907-911 (2013).

23. Patra, A. K., Dutta, A. \& Bhaumik, A. Cu nanorods and nanospheres and their excellent catalytic activity in chemoselective reduction of nitrobenzenes. Catal. Commun. 11(7), 651-655 (2010).

24. Salam, N. et al. Silver nanoparticles embedded over mesoporous organic polymer as highly efficient and reusable nanocatalyst for the reduction of nitroarenes and aerobic oxidative esterification of alcohols. Appl. Catal. A 477, 184-194 (2014).

25. Su, D. S. et al. Surface chemistry of Ag particles: Identification of oxide species by aberration-corrected TEM and by DFT calculations. Angew. Chem. Int. Ed. 47(27), 5005-5008 (2008).

26. Murray, C. B., Kagan, A. C. \& Bawendi, M. G. Synthesis and characterization of monodisperse nanocrystals and close-packed nanocrystal assemblies. Annu. Rev. Mater. Sci. 30(1), 545-610 (2000).

27. Souza, B. S., Leopoldino, E. C., Tondo, D. W., Dupont, J. \& Nome, F. Imidazolium-based zwitterionic surfactant: A new amphiphilic Pd nanoparticle stabilizing agent. Langmuir 28(1), 833-840 (2012).

28. Tamura, M. \& Fujihara, H. Chiral bisphosphine BINAP-stabilized gold and palladium nanoparticles with small size and their palladium nanoparticle-catalyzed asymmetric reaction. J. Am. Chem. Soc. 125(51), 15742-15743 (2003).

29. Scott, R. W., Ye, H., Henriquez, R. R. \& Crooks, R. M. Synthesis, characterization, and stability of dendrimer-encapsulated palladium nanoparticles. Chem. Mater. 15(20), 3873-3878 (2003).

30. Islam, R. U. et al. Conjugated polymer stabilized palladium nanoparticles as a versatile catalyst for Suzuki cross-coupling reactions for both aryl and heteroaryl bromide systems. Catal. Sci. Technol. 1(2), 308-315 (2011). 
31. Islam, R. U., Witcomb, M. J., Scurrell, M. S., Van Otterlo, W. \& Mallick, K. In situ synthesis of a Pd-poly (1, 8-diaminonaphthalene) nanocomposite: An efficient catalyst for Heck reactions under phosphine-free conditions. Catal. Commun. 12(2), 116-121 (2010).

32. Ul Islam, R. et al. Metal-polymer hybrid material as a catalyst for the heck coupling reaction under phosphine-free conditions. Synth. Commun. 41(23), 3561-3572 (2011).

33. Bai, F., Xia, Y., Chen, B., Su, H. \& Zhu, Y. Preparation and carbon dioxide uptake capacity of N-doped porous carbon materials derived from direct carbonization of zeolitic imidazolate framework. Carbon 79, 213-226 (2014).

34. $\mathrm{Xu}$, Y. et al. Nitrogen-doped porous carbon materials generated via conjugated microporous polymer precursors for $\mathrm{CO}_{2}$ capture and energy storage. RSC Adv. 7(52), 32496-32501 (2017).

35. Gao, Y. et al. Nitrogen-doped porous carbon microspheres for high-rate anode material in lithium-ion batteries. Nanotechnology. 31(15), 155702 (2020).

36. Yang, P. et al. Facile synthesis of porous nitrogen-doped carbon for aerobic oxidation of amines to imines. Microporous Mesoporous Mater. 266, 198-203 (2018).

37. Zhang, F. et al. N-doped hierarchical porous carbon anchored tiny Pd NPs: A mild and efficient quinolines selective hydrogenation catalyst. Mol. Catal. 452, 145-153 (2018).

38. Mirhosseyni, M. S., Nemati, F. \& Elhampour, A. Pyrolysis of functional cellulose by ionothermal method to synthesis of mesoporous triazine carbon for supporting of Pd and its application. Carbohyd. Polym. 217, 199-206 (2019).

39. Cui, X. et al. Facile preparation of fluffy N-doped carbon modified with Ag nanoparticles as a highly active and reusable catalyst for catalytic reduction of nitroarenes. J. Colloid Interface Sci. 506, 524-531 (2017).

\section{Acknowledgements}

The authors appreciate partial financial supports from Alzahra University. M.M.H. is also thankful to Iran National Science Foundation (INSF) for the individual given grant.

\section{Author contributions}

N.V-N., P.M., and A.A.: Visualization, Writing original draft, Formal analysis. M.M.H.: Funding acquisition, Methodology, Supervision, Writing-review, and editing.

\section{Competing interests}

The authors declare no competing interests.

\section{Additional information}

Supplementary information is available for this paper at https://doi.org/10.1038/s41598-020-74232-4.

Correspondence and requests for materials should be addressed to M.M.H.

Reprints and permissions information is available at www.nature.com/reprints.

Publisher's note Springer Nature remains neutral with regard to jurisdictional claims in published maps and institutional affiliations.

(c) (i) Open Access This article is licensed under a Creative Commons Attribution 4.0 International License, which permits use, sharing, adaptation, distribution and reproduction in any medium or format, as long as you give appropriate credit to the original author(s) and the source, provide a link to the Creative Commons licence, and indicate if changes were made. The images or other third party material in this article are included in the article's Creative Commons licence, unless indicated otherwise in a credit line to the material. If material is not included in the article's Creative Commons licence and your intended use is not permitted by statutory regulation or exceeds the permitted use, you will need to obtain permission directly from the copyright holder. To view a copy of this licence, visit http://creativecommons.org/licenses/by/4.0/.

(C) The Author(s) 2020 